\title{
Collective Oscillations in Trapped Bose-Einstein Condensed Gases in the Presence of Weak Disorder
}

\author{
G.M. Falco, A. Pelster, R. Graham \\ Universität Duisburg-Essen, \\ Fachbereich Physik, Campus Duisburg \\ Lotharstrasse 1, 47057 Duisburg, Germany
}

\begin{abstract}
The influence of a weak random potential on the collective modes of a trapped interacting BoseEinstein condensate at zero temperature is calculated in the limit when the correlation length of the disorder is smaller than the healing length of the superfluid. The problem is solved in the ThomasFermi limit by generalizing the superfluid hydrodynamic equations to allow for the presence of weak disorder. We find that the disorder-induced frequency-shifts of the low-energy excitations can be of the same order of magnitude as the beyond mean-field corrections in the normal interaction recently observed experimentally.
\end{abstract}

\section{INTRODUCTION}

Some time ago, Huang and Meng [1] have studied a homogeneous three-dimensional hard-sphere Bose gas in a random external potential as a model of superfluid helium in a disordered medium [2]. In qualitative agreement with the experiments in porous media, they found that the formation of local condensates in the minima of the random potential reduces the superfluid component of the fluid even at zero temperature, where, in the absence of disorder, the whole fluid would be superfluid [3]. The recent experimental advances in trapping BoseEinstein condensates in a disordered medium [4, 5] makes it interesting to test in a more quantitative manner the predictions of the model considered by Huang and Meng. For this purpose, we extend the latter approach to include a harmonic trapping potential in addition to the weak external random potential. In the present work we consider a condensate in the limit of a large number of particles $N$ and in the presence of disorder with a correlation length shorter than the healing length of the superfluid. These conditions allow for a simple hydrodynamical formulation of the problem similar to the theory of wave propagation in random elastic media [ [] .

In the case of weak disorder, the corrections in the density profile or in the released energy of the HuangMeng theory [1] turn out to be too small to be measurable. Nevertheless, in the present paper we argue that disorder-induced shifts might be observable in the frequencies of the collective excitations 7], because these can be measured with an accuracy of a few fractions of a percent [8]. More precisely, we show that long wavelength disorder can shift the frequencies of the low-energy excitations with the same order of magnitude but opposite sign as the beyond mean-field shifts due to repulsive atomic interactions [9] in the gas. The latter effect has been recently observed experimentally by the Innsbruck group [10]. Furthermore, in a harmonic trap, the influences of disorder and interaction should be readily distinguishable. This follows because, according to the gen- eralized Kohn's theorem 11, 12], the interaction cannot alter the frequency of the lowest dipole mode [9] while, as we show, the latter is shifted by the presence of a weak random external potential.

\section{HYDRODYNAMIC EQUATIONS}

We consider a harmonically trapped Bose gas in an external random field. The grand-canonical Hamiltonian of the system is

$$
K=\int d \mathbf{r}\left\{\psi^{\dagger}\left[-\frac{\hbar^{2} \nabla^{2}}{2 m}-\mu+U+V_{\mathrm{ext}}\right] \psi+\frac{g}{2}{\psi^{\dagger}}^{2} \psi^{2}\right\}
$$

where $\psi^{\dagger}(\mathbf{r}), \psi(\mathbf{r})$ are the field operators of an atom with mass $m, \mu$ is the chemical potential, and the interaction at low energy is described by the two-body $\mathrm{T}$-matrix $g=4 \pi \hbar^{2} a / m$ in terms of the $s$-wave scattering length $a$. The harmonic trapping potential is $V_{\text {ext }}(\mathbf{r})=m\left(\omega_{x}^{2} x^{2}+\omega_{y}^{2} y^{2}+\omega_{z}^{2} z^{2}\right) / 2$. The oscillator frequencies define the harmonic oscillator length $a_{\mathrm{HO}}=$ $\left(\hbar / m \omega_{\mathrm{HO}}\right)^{1 / 2}$, where $\omega_{\mathrm{HO}}=\left(\omega_{x} \omega_{y} \omega_{z}\right)^{1 / 3}$. The disorder potential $U$ is chosen with a Gaussian probability distribution characterized by the ensemble averages $\langle U(\mathbf{r})\rangle=0$ and $\left\langle U(\mathbf{r}) U\left(\mathbf{r}^{\prime}\right)\right\rangle=R\left(\mathbf{r}-\mathbf{r}^{\prime}\right)$, with $R(\mathbf{r})=$ $\int d \mathbf{k} /(2 \pi)^{3} e^{i \mathbf{k r}} R(\mathbf{k})$. In the case of a fast-decaying disorder-correlation $R(\mathbf{r})$, the results of the theory do not depend significantly on its shape [13]. We consider the case of a Gaussian correlation $R(\mathbf{k})=R_{0} e^{-k^{2} \xi^{2} / 2}$, where $R_{0}$ and $\xi$ characterize the strength and the correlation length of the disorder, respectively.

A random potential in a Bose-Einstein condensate causes incoherent scattering which tends to localize and to deplete the condensate wavefunction. For a statistically homogeneous system, Huang and Meng have shown [1] that, even at $T=0$, this results also in a depletion of the superfluid density $n_{s}$. This latter must be distinguished from the condensate density $n_{0}$ and is related to the total density $n$ by the relation $n_{s}=n-n_{n}$, where the normal (i.e. non-superfluid) component of the gas $n_{n}$ 
can be related to the disorder-induced depletion $n_{\mathrm{R}}$ of the condensate through $n_{n}=(4 / 3) n_{\mathrm{R}}$ [1]. Consistently with this picture, we assume that for weak disorder and at $T=0$ the superfluid component of the gas can be described by the phenomenological two-fluid hydrodynamic equations [3]

$$
\begin{aligned}
& \frac{\partial}{\partial t} n+\nabla\left(\mathbf{v}_{s} n_{s}+\mathbf{v}_{n} n_{n}\right)=0 \\
& m \frac{\partial}{\partial t} \mathbf{v}_{s}+\nabla\left(\mu+\frac{1}{2} m \mathbf{v}_{s}^{2}\right)=0 .
\end{aligned}
$$

The hydrodynamical variables in this description are assumed to be ensemble averages over the realizations of the disorder potential. Therefore, the physical validity of Eqs. (2) must be considered as being restricted to the self-averaging regime where the wavelength $q^{-1}$ of the hydrodynamic modes is much larger than the length-scale $\xi$ of the disorder potential. This condition determines the range of validity of our theory. The equations (2) can be understood as a Landau "two-fluid" model, for the unpinned part of the condensate as superfluid component and the localized pinned condensate as normal component. We use these equations to describe the collective excitations of energy $\hbar \omega \approx \hbar \omega_{\mathrm{HO}} \ll \mu$ in the presence of the harmonic trap. In the Thomas-Fermi regime and for long-wavelength oscillations 14], the non-uniform system can be considered locally as homogeneous, with the space-time-dependent density $n(\mathbf{r}, t)$ and the chemical potential related by $\mu(\mathbf{r}, t)=\mu_{l}[n(\mathbf{r}, t)]+V_{\text {ext }}(\mathbf{r})$ in the local density approximation [14]. Here $\mu_{l}[n(\mathbf{r}, t)]$ is the chemical potential of a uniform gas at density $n(\mathbf{r}, t)$. Because of the pinned character of the normal component, we must furthermore assume that only the superfluid component reacts to the probe, while the pinned normal component remains stationary. This situation is reminiscent of the physically closely related problem of the fourth sound in ${ }^{4} \mathrm{He}[3]$ and is expressed by the condition $\mathbf{v}_{n}=0$ in Eqs. (2).

Decomposing $n(\mathbf{r}, t)=n(\mathbf{r})+\delta n(\mathbf{r}, t)$ with the timeindependent background density $n(\mathbf{r})=n_{s}(\mathbf{r})+n_{n}(\mathbf{r})$, and $\mu(\mathbf{r}, t)=\mu_{0}+\delta \mu(\mathbf{r}, t)$ with $\delta \mu=(\partial \mu / \partial n) \delta n$, and restricting ourselves to the linear regime, Eqs. (2) lead to the wave-equation

$$
m \frac{\partial^{2}}{\partial t^{2}} \delta n(\mathbf{r}, t)-\nabla\left[n_{s}(\mathbf{r}) \nabla\left(\frac{\partial \mu_{l}[n(\mathbf{r})]}{\partial n(\mathbf{r})} \delta n(\mathbf{r}, t)\right)\right]=0 .
$$

The disorder and the trap both appear implicitely in Eq. (3) via the superfluid density $n_{s}(\mathbf{r})$ and the equation of state $\mu_{l}[n(\mathbf{r})]$ which are determined in the following two sections, respectively. In the limit of a statistically uniform gas, Eq. (3) reduces to the wave-equation in a medium with a random refractive index [17.

\section{MEAN-FIELD THEORY IN THE TRAP}

In order to complete the equation of motion (3) we have to determine both the equilibrium superfluid density and the equation of state. To this purpose we now extend the Huang-Meng theory to a non-uniform system, by making use of the local density approximation. Expanding locally in plane waves $\Psi(\mathbf{r})=(1 / \sqrt{V}) \sum_{\mathbf{k}} a_{\mathbf{k}}(\mathbf{r}) e^{i \mathbf{k r}}$, the presence of the condensate is taken into account by setting $a_{\mathbf{k}}(\mathbf{r})=\left\langle\sqrt{n_{\mathbf{0}}(\mathbf{r}) V}\right\rangle \delta_{\mathbf{0}, \mathbf{k}}+\delta a_{\mathbf{k}}(\mathbf{r})$. Retaining only the quadratic terms in the excitations $\delta a_{\mathbf{k}}, \delta a^{\dagger} \mathbf{k}$ from the condensate, the truncated Hamiltonian obtained from Eq. (11) can be diagonalized by the Bogoliubov transformation [1] $\delta a_{\mathbf{k}}(\mathbf{r})=u_{\mathbf{k}}(\mathbf{r}) \alpha_{\mathbf{k}}-v_{\mathbf{k}}(\mathbf{r}) \alpha_{-\mathbf{k}}^{\dagger}-z_{\mathbf{k}}(\mathbf{r})$ and $\delta a_{\mathbf{k}}^{\dagger}(\mathbf{r})=u_{\mathbf{k}}^{*}(\mathbf{r}) \alpha_{\mathbf{k}}^{\dagger}-v_{\mathbf{k}}^{*}(\mathbf{r}) \alpha_{-\mathbf{k}}-z_{\mathbf{k}}^{*}(\mathbf{r})$, where the coherence factors $u_{\mathbf{k}}$ and $v_{\mathbf{k}}$ and the complex numbers $z_{\mathrm{k}}$ can be taken real by appropriately choosing the phase of the complex fields. Then we have $u_{\mathbf{k}}^{2}(\mathbf{r})=\left\{1+\left[\epsilon_{\mathbf{k}}-\mu+V_{\text {ext }}(\mathbf{r})+2 g n(\mathbf{r})\right] / \hbar \Omega_{\mathbf{k}}(\mathbf{r})\right\} / 2$, $v_{\mathbf{k}}^{2}(\mathbf{r})=u_{\mathbf{k}}^{2}(\mathbf{r})-1, \quad$ and $z_{\mathbf{k}}(\mathbf{r})=$ $[n(\mathbf{r}) V]^{\frac{1}{2}} U_{\mathbf{k}}\left[u_{\mathbf{k}}(\mathbf{r})-v_{\mathbf{k}}(\mathbf{r})\right]^{2} / \hbar \Omega_{\mathbf{k}}(\mathbf{r}), \quad$ with $\epsilon_{\mathbf{k}}=\hbar^{2} k^{2} / 2 m$ and, where the Bogoliubov spectrum [15] is given by $\hbar \Omega_{\mathbf{k}}(\mathbf{r})=$ $\sqrt{\left[\epsilon_{\mathbf{k}}-\mu+V_{\text {ext }}(\mathbf{r})+2 g n(\mathbf{r})\right]^{2}-[g n(\mathbf{r})]^{2}}$. The density is given by $n(\mathbf{r})=n_{0}(\mathbf{r})+n_{\mathrm{Bog}}(\mathbf{r})+n_{\mathrm{R}}(\mathbf{r})$, where, besides the condensate density, $n_{0}(\mathbf{r})$ the local Bogoliubov depletion density [15] $n_{\text {Bog }}(\mathbf{r})=(8 / 3) n(\mathbf{r}) \sqrt{n(\mathbf{r}) a^{3} / \pi}$ appears, and the depletion $n_{\mathrm{R}}(\mathbf{r})$ induced by the random potential

$$
n_{\mathrm{R}}(\mathbf{r})=n_{\mathrm{R}_{0}}(\mathbf{r}) f_{\mathrm{R}}\left[4 \pi n(\mathbf{r}) \xi^{2} a\right] .
$$

Here, $n_{\mathrm{R}_{0}}(\mathbf{r})=R_{0}\left(m^{2} / 8 \pi^{3 / 2} \hbar^{4}\right) \sqrt{n(\mathbf{r}) / a}$ denotes the local depletion in the limit of a $\delta$-correlated disorder potential and the function [16]

$$
f_{\mathrm{R}}(x)=\left[e^{2 x}(1+4 x) \operatorname{Erfc}(\sqrt{2 x})-2 \sqrt{2 x / \pi}\right]
$$

(with $f_{\mathrm{R}}(0)=1$ ) includes the effects of its non-vanishing correlation length $\xi$. With $n_{\mathrm{R}}(\mathbf{r})$ now in hand, the local superfluid density $n_{s}(\mathbf{r})$ is given in terms of $n(\mathbf{r})$ by the relations $n_{s}(\mathbf{r})=n(\mathbf{r})-n_{n}(\mathbf{r})$, where the normal component is $n_{n}(\mathbf{r})=(4 / 3) n_{\mathrm{R}}(\mathbf{r})$ as discussed before.

The total disorder-induced depletion of the condensate $N_{\mathrm{R}}=\int d \mathbf{r} n_{\mathrm{R}}(\mathbf{r})$, can be calculated to leading order in $R_{0}$ by replacing in Eqs. (4) the density $n(\mathbf{r})$ by its zero-order Thomas-Fermi approximation $n(\mathbf{r}) \simeq$ $\left[\mu_{\mathrm{TF}}-V_{\mathrm{ext}}(\mathbf{r})\right] / g$, where $\mu_{\mathrm{TF}}=\frac{\hbar \omega_{\mathrm{HO}}}{2}\left(\frac{15 N a}{a_{\mathrm{HO}}}\right)^{2 / 5}$ is the mean-field chemical potential of the Gross-Pitaevskii theory. In the case of $\delta$-correlated disorder, integrating over the mean-field Thomas-Fermi radius we get $N_{\mathrm{R}_{0}} / N \simeq(15 \pi / 32)\left[n_{\mathrm{R}_{0}}(\mathbf{0}) / n(\mathbf{0})\right]$. The theory is valid when $N_{\mathrm{R}_{0}} / N \ll 1$, which can also be rewritten as $R_{0}^{\prime}(\mathbf{r}) \equiv m^{2} R_{0} / 8 \pi^{3 / 2} \hbar^{4} \sqrt{n(\mathbf{r}) a} \ll 1$, or, in local form as the condition $n_{\mathrm{R}_{0}}(\mathbf{r}) \ll n(\mathbf{r})$. In the Thomas-Fermi 
regime the latter condition is satisfied everywhere in the trap with the exception of a narrow shell at the boundaries where the condensate density vanishes. In the present work we neglect possible small corrections from this effect.

\section{BEYOND MEAN-FIELD EQUATION OF STATE}

The ground-state density $n(\mathbf{r})$ at equilibrium in Eq. (3) can be calculated by using the local density approximation for the chemical potential $\mu_{0}=\mu_{l}[n(\mathbf{r})]+$ $V_{\text {ext }}(\mathbf{r})$. In the mean-field Huang-Meng theory outlined in the previous section, the latter is determined by the Thomas-Fermi result $\mu_{\mathrm{TF}}=g n(\mathbf{r})+V_{\text {ext }}(\mathbf{r})$ of the GrossPitaevskii theory. In this mean-field approximation the effects of quasi-particle interactions [20] as well as the scattering between the quasi-particles and the impurities [18] are neglected. In order to incorporate these processes in the calculation of the collective modes, the groundstate value of the chemical potential $\mu$ has to be fixed in terms of $N=\int d \mathbf{r} n(\mathbf{r})$ by including beyond mean-field corrections in the equation of state. These latter can be calculated in the framework of the Bogoliubov theory [19] resulting in

$$
\mu_{l}(n)=n g\left(1+\frac{32}{3} \sqrt{\frac{n a^{3}}{\pi}}\right)+\mu_{\mathrm{R}, l}(n),
$$

where $\mu_{\mathrm{R}, l}(n)=6 g n_{\mathrm{R}_{0}}(\mathbf{r}) f_{\mu}\left[4 \pi n(\mathbf{r}) \xi^{2} a\right]$, and $f_{\mu}(x)=$ $\left[e^{2 x}(3+4 x) \operatorname{Erfc}(\sqrt{2 x})-2 \sqrt{2 x / \pi}\right] / 3$. In the following we wish to focus on the effects of disorder. Therefore, in the equation of state (6) we can neglect the beyond mean-field corrections due to the normal interactions, keeping only those due to the disorder. This is possible because, although the two different corrections can be of the same order, they lead to effects which are additive to lowest order. Moreover, in an experiment one could focus on the effects of the disorder by tuning, via a Feshbach resonance, to the regime $R_{0}^{\prime}(\mathbf{0}) \gg \sqrt{n(\mathbf{0}) a^{3}}$ where they become dominant. Other corrections due to finite-size, non-linearity and temperature have been sufficiently discussed in Ref. [9] in connection with the frequency shifts induced by the beyond mean-field effects in the theory of Huang and Yang 20].

Using Eq. (6), for small disorder, we can find the equation for the ground-state density by iteration. Defining the Thomas-Fermi density as $n_{\mathrm{TF}}(\mathbf{r})=\left[\mu_{0}-V_{\text {ext }}(\mathbf{r})\right] / g$, with $\mu_{0}(N)$ determined from the normalization condition $N=\int d \mathbf{r} n(\mathbf{r})$ including the disorder correction in the equation of state (6), we find

$$
n(\mathbf{r}) \simeq n_{\mathrm{TF}}(\mathbf{r})-6 n_{\mathrm{R}_{0}}(\mathbf{r}) f_{\mu}\left[4 \pi n_{\mathrm{TF}}(\mathbf{r}) \xi^{2} a\right]
$$

In the derivation we have simplified the disorder-induced depletion $n_{\mathrm{R}_{0}}$ (r) of Eq. (4) via replacing $n$ by its zeroorder approximation $n_{\mathrm{TF}}$. The same simplification is made in the argument of $f_{\mathrm{R}}$ and will be used in that of the function $f_{\chi}$ defined below.

Another quantity we need to evaluate in the hydrodynamic equation (3) is the term proportional to the inverse of the compressibility. From Eqs. (4) and (6) we have

$$
\begin{aligned}
\left(\partial \mu_{l} / \partial n\right)= & g\left\{1+3\left[n_{\mathrm{R}_{0}}(\mathbf{r}) / n_{\mathrm{TF}}(\mathbf{r})\right] f_{\mu}\left[4 \pi n_{\mathrm{TF}}(\mathbf{r}) \xi^{2} a\right]\right. \\
& \left.+6\left[n_{\mathrm{R}_{0}}(\mathbf{r}) / n_{\mathrm{TF}}(\mathbf{r})\right] f_{\chi}\left[4 \pi n_{\mathrm{TF}}(\mathbf{r}) \xi^{2} a\right]\right\}
\end{aligned}
$$

with $f_{\chi}(x)=x\left[\frac{2}{3} e^{2 x}(5+4 x) \operatorname{Erfc}(\sqrt{2 x})-\frac{4}{3} \sqrt{\frac{2}{\pi}} \frac{1+x}{\sqrt{x}}\right]$.

\section{COLLECTIVE MODES}

Having calculated the beyond mean-field corrections due to disorder in the equation of state, we can proceed to determine the explicit form of the hydrodynamic equation describing the low-energy collective modes of the system in the linear regime. Using the result of Eq. (7) and Eq. (8), and retaining only terms linear in $R_{0}$, the hydrodynamic equation of Eq. (3) can be put into the final form

$$
\begin{gathered}
m \frac{\partial^{2}}{\partial t^{2}} \delta n-\nabla\left[g n_{\mathrm{TF}} \nabla \delta n\right]=-\nabla\left[\frac{4 n_{\mathrm{R}_{0}} f_{\mathrm{R}}}{3} g \nabla \delta n\right] \\
-\nabla^{2}\left\{g\left[3 n_{\mathrm{R}_{0}} f_{\mu}-6 n_{\mathrm{R}_{0}} f_{\chi}\right] \delta n\right\}
\end{gathered}
$$

where we have left the $\mathbf{r}$-dependence of the coefficients implicit. In the limit of a uniform gas the solutions of Eq. (9) exhibit a phonon dispersion $\hbar \omega=$ $c q$. Using Eq. (17) and expanding for $\xi \ll \xi_{\text {heal }}$ we find $c^{2} \simeq c_{0}^{2}\left[1+\left(\frac{5}{3}-\frac{32}{3 \sqrt{\pi}} \frac{\xi}{\xi_{\text {heal }}}\right) \frac{n_{\mathrm{R}}}{n}\right]$ which describes the shift of the velocity of sound induced by the disorder from the Bogoliubov result $c_{0}^{2}=g n / m$ of the clean system with healing length $\xi_{\text {heal }}=1 / \sqrt{8 \pi n a}$. When $\xi=0$ this reproduces the result found in Refs. 18, 21] for $\delta$-correlated disorder. Moreover, putting the r.h.s. of Eq. (9) equal to zero we recognize the mean-field equation used by Stringari in Ref. [7] in the case of an isotropic trap. This yields the dispersion relation $\omega_{0}\left(n_{r}, l\right)=\omega_{\mathrm{HO}}\left(2 n_{r}^{2}+2 n_{r} l+3 n_{r}+l\right)^{1 / 2}$, for excitations with $n_{r}$ radial nodes and $l$ angular momentum. In the presence of disorder, Eq. (9) can be solved considering the right-hand side as a small perturbation. Defining the function $h(\mathbf{r})=3 n_{\mathrm{R}_{0}}(\mathbf{r}) f_{\mu}\left[4 \pi \xi^{2} n_{\mathrm{TF}}(\mathbf{r}) a\right]-$ $6 n_{\mathrm{R}_{0}}(\mathbf{r}) f_{\chi}\left[4 \pi \xi^{2} n_{\mathrm{TF}}(\mathbf{r}) a\right]$, we find ultimately the frequency shift

$$
\frac{\delta \omega_{0}(\xi)}{\omega_{0}}=\frac{g}{2 m \omega_{0}^{2}} \frac{\int d \mathbf{r}\left[\left(\nabla^{2} \delta n^{*}\right) h \delta n+\delta n^{*} \nabla\left(\frac{4}{3} n_{\mathrm{R}_{0}} f_{\mathrm{R}} \nabla \delta n\right)\right]}{\int d \mathbf{r} \delta n^{*} \delta n},
$$

where $\delta n$ are the solutions of the mean-field equation. In contrast with the beyond mean-field effects due to the 
atomic interaction described in Ref. [9], the frequencies of the so-called surface-modes are influenced by disorder. These modes have the principal quantum number $n_{r}=0$ and represent solutions of the type $\delta n(\mathbf{r}) \sim r^{l} Y_{l m}$ with a mean-field dispersion law given by [7] $\omega_{0}\left(n_{r}=0\right)=$ $\omega_{\text {HO }} \sqrt{l}$. Because they satisfy the condition $\nabla^{2} \delta n(\mathbf{r})=0$ the left term in the numerator of the r.h.s. of Eq. (10) is zero. Note that this term comes from the change in the macroscopic compressibility contained in the term $\partial \mu / \partial n$ in the equation of state of Eq. (3). However, the right term carrying the factor $4 / 3$ does not vanish. This correction originates because the normal part of the liquid remains stationary under an external probe. In general we obtain for the frequency shift of the surface modes

$$
\frac{\delta \omega_{0}(\xi)}{\omega_{0}}=\frac{2 l+3}{3} R_{0}^{\prime}(\mathbf{0}) I_{0, l, m}(\tilde{\xi})
$$

with

$I_{0, l, m}(\tilde{\xi})=2 \int_{0}^{1} d \tilde{r} \tilde{r}^{2 l+1} \frac{\partial}{\partial \tilde{r}}\left[\left(1-\tilde{r}^{2}\right)^{1 / 2} f_{\mathrm{R}}\left(\tilde{\xi}^{2}\left(1-\tilde{r}^{2}\right)\right)\right]$

independent from the quantum number $m$. The dimensionless variable $\tilde{\xi}$ is given by the relation $\tilde{\xi}^{2}=$ $\left(\xi R_{\mathrm{TF}} / a_{\mathrm{HO}}^{2}\right)^{2} / 2$. In the case of the dipole mode $\delta n \sim$ $r \cos \theta$ with $l=1, m=0$, the mean-field result $\omega_{\text {dip }}=\omega_{\mathrm{HO}}$ coincides with the harmonic oscillator result $\omega_{\text {osc }}=\omega_{\text {HO }}\left(2 n_{r}+l\right)$. This follows from the fact that in a harmonic potential the lowest dipole mode $\left(n_{\mathrm{r}}=0\right.$, $l=1$ ) corresponds to the oscillation of the center of mass, and is unaffected by the interatomic forces (Kohn's theorem) 11]. However, during its motion, the superfluid density is "hampered" by the normal component which remains stationary. In the limit of $\delta$-correlated disorder, Eq. (11) gives $\delta \omega_{\text {dip }}(\xi=0) / \omega_{\text {dip }}=-(5 \pi / 16) R_{0}^{\prime}(\mathbf{0})$. In the same limit, the shift of the quadrupole mode $l=2, m=2$, described by $\delta n \sim r \sin ^{2} \theta e^{2 i \phi}$, is $\delta \omega_{\mathrm{Q}}(\xi=0) / \omega_{\mathrm{Q}}=-(35 \pi / 96) R_{0}^{\prime}(\mathbf{0})$. According to the definition of the dimensionless parameter $R_{0}^{\prime}(\mathbf{0})$, we see that, besides disorder, also the presence of interactions and thus of superfluidity, is crucial in order to have the effect.

The effects of a non-zero disorder correlation length $\xi$ on the frequency shift of the surface modes can be better understood when considering the ratio

$$
\frac{\delta \omega_{0}(\xi)}{\delta \omega_{0}(0)}=-\frac{2}{\sqrt{\pi}} \frac{\Gamma(l+2)}{\Gamma(l+3 / 2)} I_{0, l, m}(\tilde{\xi})
$$

obtained from Eq. (11). In Fig. 1 the result is illustrated in the case of the dipole and the quadrupole oscillations. We plot the relative frequency shift $\delta \omega_{0}(\xi) / \delta \omega_{0}(0)$ as a function of the dimensionless variable $\tilde{\xi}=\xi R_{\mathrm{TF}} / a_{\mathrm{HO}}^{2} \sqrt{2}$ in the case of the surface excitations with $l=1$ and $l=2$. The shift decays rapidly with increasing $\tilde{\xi}$. However, as we have anticipated above, the approximation

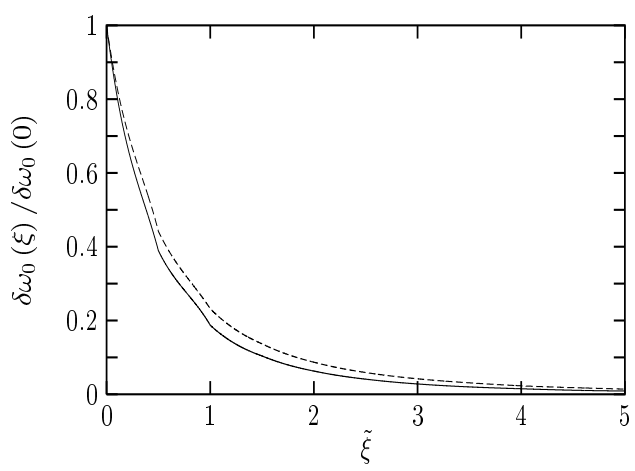

Figure 1: Relative frequency shift $\delta \omega_{0}(\xi) / \delta \omega_{0}(0)$ as a function of the variable $\tilde{\xi}=\xi R_{\mathrm{TF}} / a_{\mathrm{HO}}^{2} \sqrt{2}$ in the case of the surface excitations with $l=1$ (solid line) and $l=2$ (dashed line).

introduced in order to derive the hydrodynamic equation (9), limits the range of validity of our theory to random potentials with coherence length $\xi$ much smaller than the healing length $\xi_{\text {heal }}=1 / \sqrt{8 \pi n(\mathbf{0}) a}$ of the superfluid. By using the relation between the central density $n(\mathbf{0})$ and the Thomas-Fermi radius we have that $\xi_{\text {heal }}=R_{\mathrm{TF}}\left(a_{\mathrm{HO}} / R_{\mathrm{TF}}\right)^{2}$ and the inequality $\xi \ll \xi_{\text {heal }}$ can thus be rewritten as $\sqrt{2} \tilde{\xi} \ll 1$. Therefore, the results of Fig. 1 for the region $\sqrt{2} \tilde{\xi} \gtrsim 1$ can only be considered as an extrapolation.

Experimentally, the effects of a random potential on the dipole and quadrupole modes of a trapped BoseEinstein gas have been investigated in Ref. [4] by using optical laser speckles as realizations of disorder configurations. In these experiments, the smallest length scale of the speckle potential is of about $\xi \simeq 10 \mu \mathrm{m}$ while the axial Thomas-Fermi radius is $100 \mu \mathrm{m}$. As a typical experimental situation where the Thomas-Fermi condition $N a / a_{\mathrm{HO}} \gg 1$ is satisfied, we can assume $R_{\mathrm{TF}} / a_{\mathrm{HO}} \simeq 10$. This would imply a value $\tilde{\xi} \simeq 6$ in our description, which is far beyond its range of validity. In particular, for large values of the disorder correlation length, such that $\xi \gtrsim \xi_{\text {heal }}$, the system is not self-averaging and the observed frequency-shifts must depend on each individual realization of the speckle potential [22, 23]. In that case, the disorder average must experimentally be taken by determining, for each mode, the mean value of the measured frequencies over many different realizations. Both experiment [4] and theory 22] predict no shift in that regime in qualitative agreement with our extrapolation. Decreasing further the correlation length of the disorder would allow to enter the regime $\xi \ll \xi_{\text {heal }}$, where the system becomes self-averaging, and the shift predicted in our theory should become observable.

Differently than for the surface modes, the meanfield frequency $\omega_{\mathrm{M}}=\sqrt{5} \omega_{\mathrm{HO}}$ of the lowest compres- 
sional mode $\left(n_{r}=1, l=0\right)$ is shifted by the beyond mean-field corrections in the normal interaction. The shift has been calculated in Ref. [9] and is $\delta \omega_{\mathrm{M}} / \omega_{\mathrm{M}}=$ $(63 \pi / 128) \sqrt{a^{3} n(\mathbf{0})}$. Inserting the density oscillation $\delta n \propto 1-(5 / 3) r^{2}$ the analogous correction due to the disorder calculated from Eq. (10) in the limit $\xi=0$ amounts to $\delta \omega_{\mathrm{M}} / \omega_{\mathrm{M}}=-(469 \pi / 768) R_{0}^{\prime}(\mathbf{0})$.

\section{ANISOTROPIC TRAP}

The results can be generalized to non-spherical traps, by considering an axially deformed trap of the form $V_{\text {ext }}(\mathbf{r})=m\left(\omega_{\perp}^{2} r_{\perp}^{2}+\omega_{z}^{2} z^{2}\right) / 2$, where $r_{\perp}=\sqrt{x^{2}+y^{2}}$ is the radial coordinate. In order to put better in evidence the role of the anisotropy, we restrict ourselves to the case of disorder with vanishing correlation length. In the presence of anisotropy, the functions of the form $\delta n \sim r^{l} Y_{l m}$ are still solutions of the l.h.s. of Eq. (9). In the case of the dipole oscillation $(l=1, m=0)$ with the mean-field mode frequency $\omega_{\text {dip }}=\omega_{z}$, the disorder corrections lead to the same relative shift $\delta \omega_{\text {dip }} / \omega_{\text {dip }}$ as in the case of the isotropic trap. The same argument applies to the quadrupole mode $l=2, m=2$, with $\omega_{\mathrm{Q}}=\sqrt{2} \omega_{\perp}$. The quadrupole mode with $m=0$ involves a mixing with the monopole mode $n_{r}=1, l=0$. In this case the dispersion law is [7] $\left[\omega_{0}^{ \pm}(m=0)\right]^{2}=\omega_{\perp}^{2}(2+$ $\left.\frac{3}{2} \lambda^{2} \pm \frac{1}{2} \sqrt{9 \lambda^{4}-16 \lambda^{2}+16}\right)$, where $\lambda=\omega_{z} / \omega_{\perp}$ characterizes the deformation of the trap. Using the corresponding oscillation $\delta n \sim-\frac{2 \mu_{0}}{m \omega_{\perp}^{2}}\left[\left(\frac{\omega_{0}^{ \pm}}{\omega_{\perp}}\right)^{2}-2\right]+r_{\perp}^{2}+\left[\left(\frac{\omega_{0}^{ \pm}}{\omega_{\perp}}\right)^{2}-4\right] z^{2}$ in Eq. (10) we find the shifts

$$
\frac{\delta \omega_{0}^{ \pm}}{\omega_{0}^{ \pm}}=-7 \pi R_{0}^{\prime}(\mathbf{0}) \frac{ \pm 72 \pm 9 \lambda^{2}+107 \sqrt{16-16 \lambda^{2}+9 \lambda^{4}}}{1536 \sqrt{16-16 \lambda^{2}+9 \lambda^{4}}} .
$$

Note that for a spherical trap $(\lambda=1)$ we have $\omega_{0}^{+}=\sqrt{5} \omega_{\mathrm{HO}}$ and $\omega_{0}^{-}=\sqrt{2} \omega_{\mathrm{HO}}$ and Eq. (13) recovers the shift of the quadrupole mode $\delta \omega_{\mathrm{Q}} / \omega_{\mathrm{Q}}=$ $-(35 \pi / 96) R_{0}^{\prime}(\mathbf{0})$ and the shift of the monopole mode $\delta \omega_{\mathrm{M}} / \omega_{\mathrm{M}}=-(469 \pi / 768) R_{0}^{\prime}(\mathbf{0})$ calculated above. The $\lambda$-dependence of the frequency shifts of Eq. (13) is shown in Fig. 2 by plotting the functions $\delta \omega_{0}^{+}(\lambda) / \delta \omega_{\mathrm{M}}$ (lower curve) and $\delta \omega_{0}^{-}(\lambda) / \delta \omega_{\mathrm{Q}}$ (upper curve).

\section{CONCLUSIONS AND OUTLOOK}

We have calculated the shifts to the collective frequencies of a zero-temperature trapped Bose gas induced by a weak external random potential with a correlation length smaller than the healingh length of the superfluid. We have shown that the realization of such a limit in current experiments in trapped Bose-Einstein condensates in random media would allow to measure for the first time the predictions of the Huang and Meng theory in

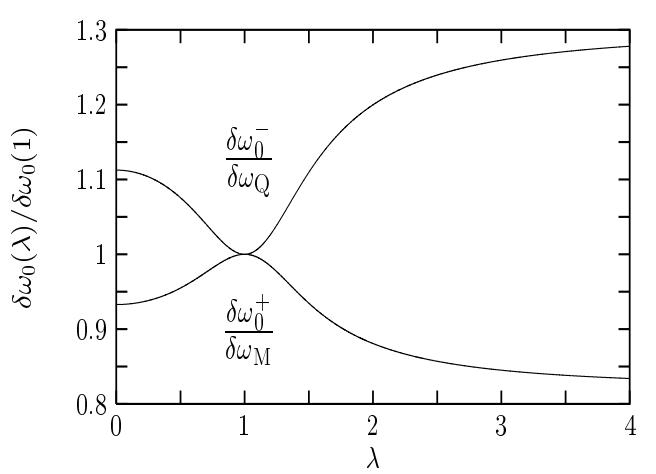

Figure 2: Relative frequency shift $\delta \omega_{0}^{+}(\lambda) / \delta \omega_{\mathrm{M}}$ (lower curve) and $\delta \omega_{0}^{-}(\lambda) / \delta \omega_{\mathrm{Q}}$ (upper curve) of the $m=0$ modes, resulting from the coupling between the quadrupole and monopole modes, as a function of the deformation parameter $\lambda=$ $\omega_{z} / \omega_{\perp}$. The disorder correlation length is taken here $\xi=0$.

a quantitative way. Moreover, the interplay between the pinned and the unpinned components of the condensate is expected to produce a deviation from the generalized Kohn's theorem for the center of mass motion of the superfluid. This latter phenomenon provides an unambiguous signature of the disorder-induced effects against the beyond mean-field corrections due to the interatomic interactions.

These results have been derived by means of a hydrodynamic approach and could be extended to consider the strongly anisotropic traps which realize the so-called "one-dimensional mean-field" regime described in Ref. 24]. This would be relevant in connection with the recent experimental and theoretical investigations of the transport of Bose-Einstein condensates in one-dimensional microtraps in the presence of disorder [25, 26, 27, 28, 29].

We thank P. Navez, J. Anglin, H. T. C. Stoof, P. Schlagheck and S. Stringari for useful discussions. This work was supported by the German DFG Research Program SFB/TR 12.

[1] K. Huang and H. F. Meng, Phys. Rev. Lett. 69, 644 (1992).

[2] M. H. W. Chan, K. I. Blum, S. Q. Murphy, G. K. S. Wong, and J. D. Reppy, Phys. Rev. Lett. 61, 1950 (1988), G. K. S. Wong, P. A. Crowell, H. A. Cho, and J. D. Reppy, Phys. Rev. Lett. 65, 2410 (1990).

[3] I. M. Kalatnikov, An introduction to the theory of superfluidity (W. A. Benjamin, Inc., New York, 1965).

[4] J.E. Lye, L. Fallani, M. Modugno, D.S. Wiersma, C. Fort, and M. Inguscio, Phys. Rev. Lett. 95, 070401 (2005).

[5] D. Clement, A. F. Varon, M. Hugbart, J. A. Retter, P. Bouyer, L. Sanchez-Palencia, D. M. Gangardt, G. V. 
Shlyapnikov, and A. Aspect, Phys. Rev. Lett. 95, 170409 (2005).

[6] A. Ishimaru, Wave propagation and scattering in a random media (Academic, New York, 1978), Vols. 1 and 2.

[7] S. Stringari, Phys. Rev. Lett. 77, 2360 (1996).

[8] D. M. Stamper-Kurn, H.-J. Miesner, S. Inouye, M. R. Andrews, and W. Ketterle, Phys. Rev. Lett. 81, 500 (1998).

[9] L. Pitaevskii and S. Stringari, Phys. Rev. Lett. 81, 4541 (1998).

[10] A. Altmeyer, S. Riedl, C. Kohstall, M. J. Wright, R. Geursen, M. Bartenstein, C. Chin, J. Hecker Denschlag, and R. Grimm, Phys. Rev. Lett. 98, 040401 (2007).

[11] W. Kohn, Phys. Rev. 123, 1242 (1961).

[12] J. F. Dobson, Phys. Rev. Lett. 73, 2244 (1994).

[13] M. Timmer, A. Pelster, and R. Graham, Europhys. Lett. 76, 760 (2006).

[14] F. Dalfovo, S. Giorgini, L. P. Pitaevskii, and S. Stringari, Rev. Mod. Phys. 71, 463 (1999).

[15] N. Bogoliubov, J Phys. USSR 11, 23 (1947).

[16] M. Kobayashi and M. Tsubota, Phys. Rev. B 66, 174516 (2002).

[17] S. John, H. Sompolinsky, and M. J. Stephen, Phys. Rev. B 27, 5592 (1983).
[18] A. V. Lopatin and V. M. Vinokur, Phys. Rev. Lett. 88, 235503 (2002).

[19] G. M. Falco, A. Pelster, and R. Graham, Phys. Rev. A (in press).

[20] K. Huang and C.N. Yang, Phys. Rev. 105, 767 (1957).

[21] S. Giorgini, L. Pitaevskii, and S. Stringari, Phys. Rev. B 49, 12938 (1994).

[22] M. Modugno, Phys. Rev. A 73, 013606 (2006).

[23] R. C. Kuhn, O. Sigwarth, C. Miniatura, D. Delande, and C. A. Müller, cond-mat/0702183

[24] C. Menotti and S. Stringari, Phys. Rev. A 66, 043610 (2005).

[25] D. W. Wang, M. D. Lukin, and E. Demler, Phys. Rev. Lett. 92, 076802 (2004).

[26] T. Paul, K. Richter, and P. Schlagheck, Phys. Rev. Lett. 94, 020404 (2005).

[27] T. Paul, P. Leboeuf, N. Pavloff, K. Richter, and P. Schlagheck, Phys. Rev. A 72, 063621(2005).

[28] C. Fort, L. Fallani, V. Guarrera, J.E. Lye, M. Modugno, D.S. Wiersma, and M. Inguscio, Phys. Rev. Lett. 95, 170410 (2005).

[29] L. Sanchez-Palencia, Phys. Rev. A 74, 053625 (2006). 